\title{
Hadron calorimetry in the L3 detector
}

O. Adriani ${ }^{\text {a }}$, A. Arefiev ${ }^{\text {b }}$, Q. An ${ }^{\text {c }}$, T. Azemoon ${ }^{\text {d }, ~ T . ~ A z i z ~}{ }^{\text {e, R.C. Ball }}{ }^{\text {d }}$, S. Banerjee ${ }^{\text {e }}$, P. Blomeke ${ }^{c}$, M. Capell ${ }^{\mathrm{d}}$, X. Cai ${ }^{\text {c }}$, C. Chen ${ }^{\mathrm{g}}$, G.M. Chen ${ }^{\mathrm{g}}$, H.S. Chen ${ }^{\mathrm{g}}$, M.L. Chen ${ }^{\mathrm{d}}$, S.R. Chendvankar ${ }^{\text {e }}$, C. Civinini ${ }^{\text {a }}$, X. Cui ${ }^{\text {c }}$, R. D'Alessandro ${ }^{a}$, Yu. Galaktionov ${ }^{\text {b }}$, E. Gallo ${ }^{\text {a }}$, S.N. Ganguli ${ }^{\text {e, S. Goldfarb }}{ }^{\mathrm{d}}$, Z.F. Gong ${ }^{\mathrm{c}}$, A. Gordeev ${ }^{\mathrm{b}}$, Yu. Gorodkov ${ }^{\mathrm{b}}$, A. Gurtu ${ }^{\mathrm{e}}$, H. Haan ${ }^{f}$, H. Hofer ${ }^{\text {h }}$, M.M. Ilyas ${ }^{c}$, L.W. Jones ${ }^{\text {d }, ~ W . ~ K a r p i n s k i ~}{ }^{\text {f }}$, Yu. Kamyshkov ${ }^{b}$, R. Khan ${ }^{\text {c }}$, A. Klimentov ${ }^{b}$, O. Kornadt ${ }^{f}$, V. Koutsenko ${ }^{b}$, V. Kumar ${ }^{c}$, A. Kunin ${ }^{\text {c }}$, P. Lecomte ${ }^{h}$, P. LeCoultre ${ }^{h}$, J. Lettry ${ }^{\text {h }}$, H.T. Li ${ }^{\text {g }}$, X.G. Li ${ }^{\text {g }}$, Y.B. Lin ${ }^{\text {c }, ~ Y . S . ~ L u ~}{ }^{g}$, K. Lubelsmeyer ${ }^{\text {f }}$, A. Malinin ${ }^{\text {b }}$, P.K. Malhotra ${ }^{\text {e }}$, A. Marchionni ${ }^{\text {a }}$, K. Mazumdar ${ }^{\text {e }}$, M. Meschini ${ }^{a}$, G.B. Mills ${ }^{d}$, V. Morgunov ${ }^{b}$, L. Niessen ${ }^{f}$, D. Pandoulas ${ }^{\text {f }}$, Y.J. Pei ${ }^{f}$, M. Pieri ${ }^{a}$, V. Plyaskin ${ }^{b}$, V. Pojidaev ${ }^{b}$, K. Qureshi ${ }^{c}$, R. Raghavan ${ }^{\mathrm{e}}$, M. Rohner ${ }^{\mathrm{f}}$, B.P. Roe ${ }^{\mathrm{d}}$, S. Saran ${ }^{\mathrm{e}}$, A. Savin ${ }^{b}$, S. Shevchenko ${ }^{b}$, V. Shevchenko ${ }^{b}$, A. Schultz von Dratzig ${ }^{f}$, K. Shmakov ${ }^{b}$, E. Shoumilov ${ }^{\text {b }}$, V. Shoutko ${ }^{b}$, G. Schwering ${ }^{f}$, R. Siedling ${ }^{c}$, B. Spiess ${ }^{\text {h }}$, K. Sudhakar ${ }^{\text {e }}$, X.W. Tang ${ }^{\mathrm{g}}$, E. Tarkovsky ${ }^{\mathrm{b}}$, S.C. Tonwar ${ }^{\mathrm{e}}$, K.L. Tung ${ }^{\mathrm{g}}$, J. Ulbricht ' , I. Vetlitsky ${ }^{\mathrm{b}}$, I. Vorobiev ${ }^{b}$, M. Wadhawa ${ }^{c}$, W. Wallraff ${ }^{\text {f }}$, Y.F. Wang ${ }^{a}$, R.J. Wu ${ }^{\text {c }}$, S.W. Wu ${ }^{\text {c }}$, Y.G. Wu ${ }^{c}$, J.F. Zhou ${ }^{\mathrm{f}}$ and A. Zichichi ${ }^{\mathrm{c}}$

${ }^{a}$ INFN, Sezione di Firenze and University of Florence, Italy

${ }^{b}$ Institute of Theoretical and Experimental Physics, ITEP, Moscow, USSR

${ }^{c}$ World Laboratory, FBLJA Project, Geneva, Switzerland

${ }^{d}$ University of Michigan, Ann Arbor, USA

${ }^{e}$ Tata Institute of Fundamental Research, Bombay, India

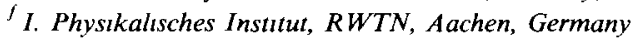

${ }^{g}$ Institute of High Energy Physics, IHEP, Belying, People's Republic of China

${ }^{h}$ Eidgenössische Technische Hochschule, ETH, Zurich, Switzerland

'Paul Scherrer Institute, PSI, Würenlingen, Switzerland

Received 12 November 1990

The characteristics of the L3 hadron calorimeter as realized in the observation of hadronic jets and other events from $\mathrm{e}^{+} \mathrm{e}^{-}$ collisions at LEP are presented and discussed. The pattern-recognition algorithm utilizing the fine granulatiry of the calorimeter is described, and the observed overall resolution of $10.2 \%$ for hadron jets from $\mathrm{Z}$ decay is reported The use of the calorimeter in providing information on muon energy losses is also noted.

\section{Introduction}

The measurement of hadronic energy in the L3 detector is carried out by combining signals from a uranıum gas proportional chamber hadron calorimeter with those of an electromagnetic detector preceeding it. An algorithm has been developed for pattern recognition and for the analysis of the energies and angles of jets produced at LEP in $\mathrm{e}^{+} \mathrm{e}^{-}$collisions at the $\mathrm{Z}^{0}$ energy, and coefficients have been obtained which optimize the resolution of the combined e.m. and hadronic detectors and provide absolute normalization. This al- gorithm is described, following a brief summary on the L3 calorimeters and a presentation of the calibration and normalization procedures. A pattern-recognition algorithm relevant to the analysis of other types of $Z^{0}$ decays (e.g. to muons) is also discussed.

The L3 detector [1] consists (from the interaction vertex outward) of a central precision tracking chamber, a bismuth germanate (BGO) crystal matrix electromagnetic calorimeter, a hadron calorimeter consisting of uranium absorber with proportional wire chamber (PWC) readout, an active muon filter, and precision muon-tracking drift chambers. The BGO crystals pro- 
vide a luminosity monitor for eletrons scattered by less than $4^{\circ}$. The entire detector is contained within a large solenoidal magnet providing a $0.5 \mathrm{~T}$ axial magnetic field.

\section{The $\mathbf{L} 3$ calorimeter}

The major components of the $\mathrm{L} 3$ calorimeter are the electromagnetic BGO matrix and the uranium gas PWC hadron calorimeter (HC). The $\mathrm{HC}$ is mechanically divided into a barrel, two end caps, and a muon filter. The BGO (for the 1989-1990 data presented here) is in a barrel extending over $42.4^{\circ} \leq \theta \leq 137.6^{\circ}$ in polar angle $\theta$ (fig. 1). A counter containing interleaved lead and scintillator sheets is located in front of the $\mathrm{HC}$ end caps.

The HC barrel consists of 144 modules grouped into 9 rings of 16 modules each. A module contains PWCs interleaved with $5 \mathrm{~mm}$ uranium plates; the 3 central rings contain 60 chambers and 59 plates, the 6 outer rings contain 53 chambers. The anode wires in adjacent PWC planes are oriented at right angles, alternately in the $z$ and $\phi$ directions. The $z$ direction is the beam direction and $\phi$ is the angle in a plane perpendicular to the $z$ direction. The signal wires are grouped into readout cells, or "towers"; each tower includes only wires from alternate, parallel layers. The pattern of wire groupings in both dimensions provides 10 towers in depth (radially), and 9 in the other dimension ( $z$ or $\phi$ ). In the $r-\phi$ plane, the towers subtend about $2.5^{\circ}$ from the interaction vertex, whereas in the $r-z$ plane they have a constant width of about $5 \mathrm{~cm}$, also corresponding to about $2.5^{\circ}$ in the central region.

Each of the two $\mathrm{HC}$ end caps (upstream and downstream) is constructed of one outer and two inner rings (fig. 1); for mechanical reasons, each ring is in turn divided vertically into two half-rings. The forward part of the outer ring $(\approx 1.3 \lambda)$ has the same sampling as the $\mathrm{HC}$ barrel ( $5 \mathrm{~mm}$ absorber). All other parts of the $\mathrm{HC}$ end caps have a sampling that is twice as coarse $(10 \mathrm{~mm}$ absorber). The chamber wires are stretched azimuthally, thus measuring the polar angle $\theta$ directly. Alternate chambers layers are oriented at $22.5^{\circ}$ with respect to each other to provide two stereo views. As in the barrel, the tubes are grouped into readout towers for each view separately. The towers are projective, pointing to the interaction vertex with an angular segmentation of about $1^{\circ}$ in $\theta$. The segmentation in azimuth is $22.5^{\circ}$, defined by the chamber dimensions. The longitudinal segmentation (in $z$ ) is 5 for the inner rings and 7 for the outer rings.

The $\mathrm{HC}$ barrel subtends a polar angle $\theta$ between $25^{\circ}$ and $155^{\circ}$, thus overlapping the $\mathrm{HC}$ end caps, which cover polar angles between $5.5^{\circ}$ and $35^{\circ}$, and between $145^{\circ}$ and $174.5^{\circ}$.

The muon filter (MF), radially just outside the $\mathrm{HC}$ barrel, subtends about $85 \%$ of $4 \pi$ in azimuth. The primary functions of the MF are to track muons between the $\mathrm{HC}$ barrel and the muon drift chambers and to absorb the tail of the hadron showers.

The BGO barrel consists of 7680 crystals, each in the form of a truncated pyramid whose axis is radial from the interaction vertex. The pyramids are $24 \mathrm{~cm}$ long

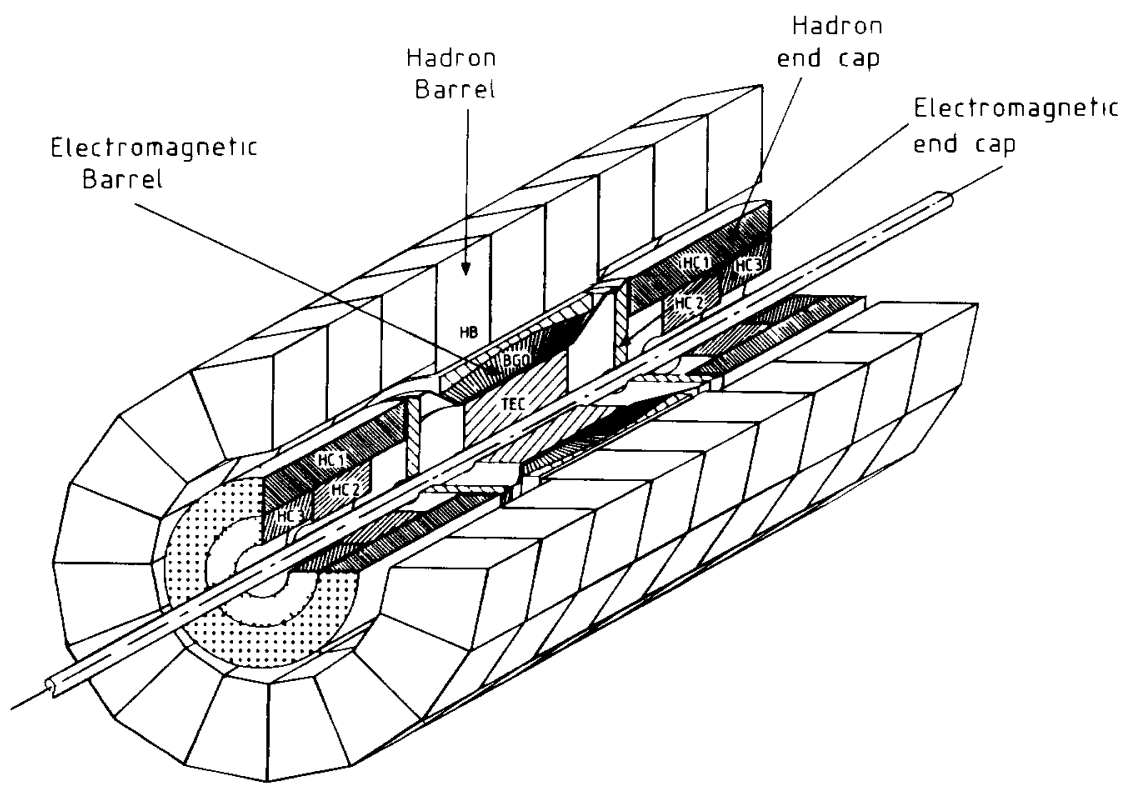

Fig. 1. Side view of the $\mathrm{L} 3$ detector calonmeter part. 
(21.4 radiation lengths, or 1.1 nucleon interaction lengths) and (about) $3 \times 3 \mathrm{~cm}^{2}$ tapering to (about) $2 \times 2$ $\mathrm{cm}^{2}$. The matrix contains 48 crystals in $\theta$ and 160 in $\phi$, corresponding to a granularity of about $2^{\circ} \times 2^{\circ}$. The BGO has a measured resolution of about $4 \%$ for 150 $\mathrm{MeV}$ electrons and better than $1 \%$ for electrons of over $4 \mathrm{GeV}$.

For the 1989-1990 running periods, a set of e.m. counters (veto counters) were installed in front of the hadron calorimeter end caps, each consisting of 12 layers of plastic scintillator interleaved with $3 \mathrm{~mm}$ thick lead plates (for a total of 6.5 radiation lengths). The counters were azimuthally segmented into 16 modules, each read out by Hamamatsu type R2490-01 photomultipliers, via wavelength shifters. These counters covered polar angles of $7^{\circ}-37^{\circ}$ and $143^{\circ}-173^{\circ}$.

\section{Gain calibration}

In order to obtain a uniform response of the different channels (towers), pedestal levels and intercalibrations were periodically checked. The towers were read out through LeCroy Fastbus model 1882F ADCs, 12-bit for the $\mathrm{HC}$ barrel and the MF, and 15-bit for the $\mathrm{HC}$ end caps. The pedestal levels could be subject to occasional drifts, and were updated daily. Pedestals from the most recent pedestal run were stored and used for subsequent data analysis, until the next pedestal run.

The intercalibration of the $\mathrm{HC}$ channels is done using the gamma signals from uranium in the PWCs. It has been found that the $U$ noise spectrum with random gating has a unique shape, which can be fitted to obtain a system gain (chamber and amplifier) for each channel with an accuracy of better than $2 \%$ [2]. Periodically (approximately once a month) a $U$ calibration run is taken so that the gain of each channel can be compared with a high-statistics reference spectrum. The distribution of the gains of the 22000 channels in the HC barrel before correction is illustrated in fig. 2. Similar results are obtained for the 3960 end cap channels. The gain depends on the chamber high voltage, the gas density, the gas composition, and the amplifier gan. The high voltage and chamber gas are continuously monitored and held constant to within about $0.1 \%$; no drift of the amplifiers is observed. The long-term stability of the HC system is found to be better than 1.3\%. The intercalibration constants are applied during off-line reconstruction to the amplitude measured in the corresponding readout channels.

It was found [3] that the response of the PWCs to the uranium radiation depends weakly on the L3 magnetic field, and is different when it comes from chambers with anode wires parallel to this field or perpendicular to it. Consequently U noise calibrations were done both with and without magnetic filed, so that the effect

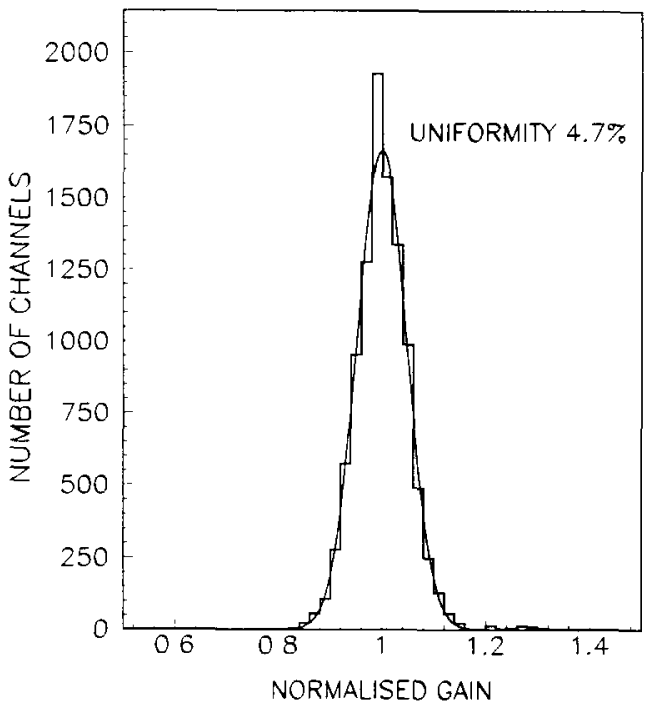

Fig. 2. Distribution of normalızed individual cell amplitudes in the uranium hadron calorimeter.

of the field on the calibration is known. On the other hand, we see no effect of the field on the signals from hadron jets. This was determined by comparing signals from both hadrons and muons before and afer applying the field, and also by comparing the signals from the two orthogonal sets of wires from the same hadron jets. The procedures described below are all carried out with the magnetic field on.

The U noise pulses correspond to a signal per channel equivalent to about $2 \mathrm{MeV}$ rms hadron energy deposition for the largest tower (including 26-28 PWC anode wires), or about $150 \mathrm{MeV}$ rms when summing over an entire module. During normal data takıng, the channels were read out only if their signal was above a given threshold, which corresponded, for the $\mathrm{HC}$, to a hadronic energy of $9 \mathrm{MeV}$. This reduces the number of uranium-induced signals recorded with each event, per HC barrel module, to about 4 out of a total of 180 channels. It should be noted that a minimum-ionizing particle (e.g. a muon) produces in each calorimeter plane a signal corresponding to about $25 \mathrm{MeV}$ of equivalent hadron energy.

The noise level in the BGO readout is also reduced by imposing a threshold of $2 \mathrm{MeV}$. The BGO readout electronics and digitization is quite different from and independent of the hadron calorimeter system, and is described elsewhere [1]. The dynamic range of the readout, for signals in each crystal ranging from less than $100 \mathrm{eV}$ to about $200 \mathrm{GeV}$, is equivalent to that of a 21-bit ADC. 


\section{Pattern recognition}

The hadronic or electromagnetic cascade initiated by a single particle or by a jet of particles must be recognized from its signature in the $\mathrm{HC}$ and $\mathrm{BGO}$. Of interest here are hadronic jets from quarks and gluons, electrons, gammas, muons, and single hadrons. The event reconstruction proceeds in two steps. In the first step the event is reconstructed in each subdetector separately. In the second step it is reconstructed globally, using the results of the first step.

The first step identifies the "hits" in the calorimeter, where a hit is a tower (channel) with over $9 \mathrm{MeV}$ energy loss in the $\mathrm{HC}$ or $2 \mathrm{MeV}$ in the $\mathrm{BGO}$, i.e. above threshold. The hit coordinate is defined as the geometrical centre of the tower or BGO crystal. "Clusters" are then formed from these hits. A cluster is defined as two or more adjacent or contıguous hits. Two hits touching at their corner are understood to be contiguous; also, in the $\mathrm{HC}$ barrel, two hits which are separated by a tower, but lie along the same radical vector from the interaction vertex, may form or be part of a cluster. This definition of a cluster strongly discriminates between particle tracks or cascades and random background, while accommodating fluctuations in the cascade process as well as possible inefficiencies in the detector coverage. As an example, a $45 \mathrm{GeV}$ jet from $Z^{0}$ decay contains about 70 hits in the $\mathrm{HC}$ barrel in a volume comparable to one module, whereas the noise level corresponds to about 4 hits (as noted above). The density of hits in clusters from muons and electrons is greater than in those from hadrons; for muons the clusters essentially define the track, and for electrons the cascade ionization density is high because of the short radiation length of uranium.

Each cluster is characterized by its type, energy, and direction. The direction is defined as the vector sum of all the hits it contains (where the vector origin is the interaction vertex). Two types of clusters are distinguished, hadronic and electromagnetic, according to their transverse and longitudinal profile. The global reconstruction procedure then defines "objects" in the detector corresponding to hadron jets, single electrons, and single muons. At this stage of the analysis, the information about muons reconstructed in the muon chambers is added to the first-step calorimeter reconstruction information.

The global reconstruction algorithm starts from the most energetic cluster, taken as the "seed" of an "object". An iteractive procedure then adds to this seed all clusters in a $30^{\circ}$ cone around the seed axis (the vector to the interaction vertex). An object axis is then defined by calculating the energy-weighted vector sum of all included cluster vectors, and a new $30^{\circ}$ cone is defined about this axis. The clusters within this newly defined cone are used to define, in turn, a new object axis. This procedure stops when no new clusters are added to the object after redefinition of its axis. Then, starting from the next most energetic cluster not included in any previously defined object, the procedure is repeated to build up a new object. This tterative reconstruction procedure stops when the energy of the next new object is less than $7 \mathrm{GeV}$. Each one of the remainıng (lowerenergy) clusters is then added to the closest existing object if it is not separated by more than $20^{\circ}$ from the closest cluster in this object. As a last step the identified objects are classified and their energies are determined.

The reconstructed object classification designates the energy calibration for the hits of this object. Thus, for a muon inside the object we take 1 ts momentum as defined by the muon chambers. The cluster and object defintions are applied to the hadron calorimeter and to the BGO signals. The object thus includes, for example, both the electromagnetic and hadronic components of a jet (as well as possible muons).

It should be noted that the L3 Collaboration has employed a procedure whereby the analysis is carried out using two independent algorithms applied in parallel. The procedure described above is that employed by one of the two; the other algorithm uses a procedure that is similar in concept but different in detail from this. The resulting physics conclusions from the two algorithms are in agreement with each other.

\section{Energy calibration}

During the course of the design and construction of the L3 experiment, calonmeter modules were tested, as prototypes and final assemblies, in beams of hadrons, electrons, and muons; results of these studies have been published [2-4]. The measured energy resolution of the $\mathrm{HC} / \mathrm{BGO}$ system for hadrons was found to $\mathrm{f}_{1 \mathrm{t}}$ $55 \% / \sqrt{E}+5 \%$, where $E$ is in $\mathrm{GeV}$. These studies provided useful orientation for the intial setting of voltages, gains, dynamic range, etc., of the calorimeters. However the results cited below are substantially independent of these earlier studies.

The $Z^{0}$ usually decays through the two-quark finalstate channel, giving rise to two back-to-back hadron jets. This in turn has provided a conventent source of objects of known energy for the calibration of the calorimeter. Furthermore, a well tuned Monte Carlo has been developed by the Lund group [5], which has been verified in $\mathrm{Z}$ decays and lower-energy electron-positron experiments. The characteristics of the cascade initiated in a particular calonmeter structure are also well understood, and are successfully simulated by the computer program GEANT [6]. Hence, the expected characteristics of jets from $Z$ decay can be and have been studied through extensive Monte Carlo runs. 
It is known, for example, that the charged hadron multiplicity is about 11 per jet, and that the average hadron energy in such jets is about $3 \mathrm{GeV}$.

In order to measure the total energy one has to add up the energy deposition in each subdetector properly. The summing algorithm should provide a uniform response with optimum energy resolution over the entire solid angle covered by the calorimeters. This is done by expressing the total energy as a linear combination of the signals recorded by each subdetector, with each signal weighted by an appropriate energy-independent conversion coefficient. These coefficients depend on the particular subdetector and on the cluster position in it. The objective of the energy calıbration is to determine the value of the coefficients which provide uniform energy response and which minimize the error in the energy determination.

The L3 hadron calorimeter system is divided into four sectors in polar angle $\theta$ in each hemisphere (forward and backward), which are characterized by different geometries, as seen in fig. 3. Thus there are eight subdetectors for which to fix parameters. The central region is further divided into four angular intervals (between $42.4^{\circ}$ and $90^{\circ}$, and correspondingly between $90^{\circ}$ and $137.6^{\circ}$ ) to seek any finer variation of coefficients withın this largest sector. The major $\theta$ dependence of the $\mathrm{HC}$ barrel response is due to the change of the effective sampling thickness with $\theta$. To compensate for this and reduce the dependence of weighting factors on $\theta$, an empirical weighting factor of $\sec \left[\left(90^{\circ}-\theta\right) / 1.5\right]$ was applied to all cluster energies in the $\mathrm{HC}$ barrel.

The callbration is done by scanning the detector with two back-to-back jets (or electrons) of known (beam) energy and with an event thrust polar angle $\theta$ between $5.5^{\circ}$ and $174.5^{\circ}$, i.e. withın the $\mathrm{HC}$ acceptance. The selection of events for this calibration is made using the selection criteria described elsewhere [7], extended to include Bhabha events. Only events with two energetic objects, each with energy greater than $7 \mathrm{GeV}$ and a

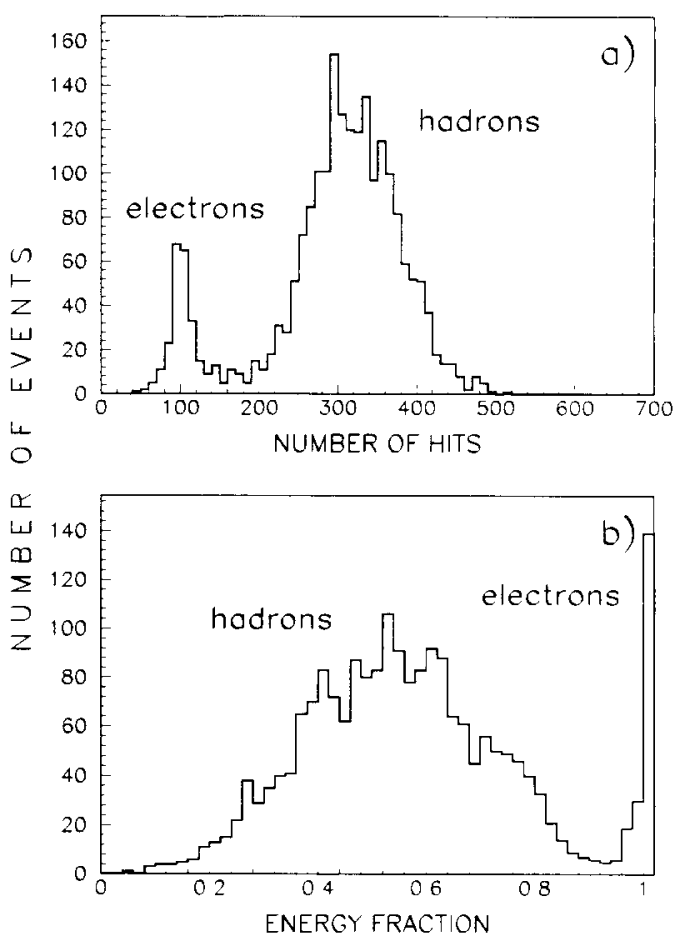

Fig. 4 Distribution of the number of hits (a), and fraction of total energy, deposited in the front-end (BGO) calorimeter part (b) for events selected for calibration.

departure from collinearity of less than $14^{\circ}$, and with both objects (jets) falling in the same $\theta$ region are used for calibration.

The energy of each event is calculated as

$E_{\text {meas }}=\sum_{i} E_{i}(\theta) W_{i}(\theta), \quad \imath=1$ to 8 .

Here $\theta$ is the polar angle of the event thrust axis, $E_{l}$ is the response, i.e. the sum of the energies of the clusters, associated with jets (or electrons) in the $\imath$ th subdetector,

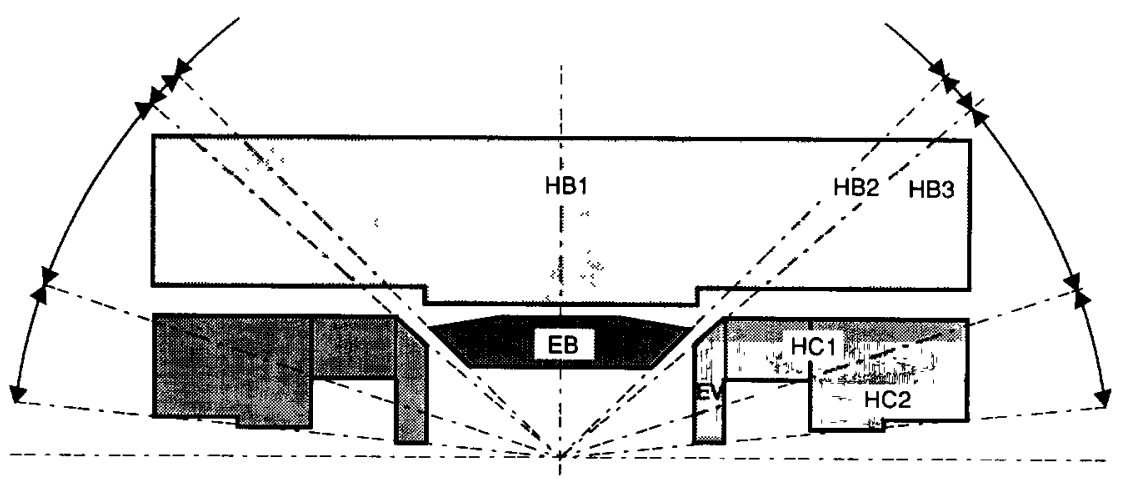

Fig. 3 Logical subdetectors used in the L3 calorimeter calibration. HB1 is the central part of the hadron calorimeter barrel; EB is the electromagnetıc calorimeter barrel (BGO crystals array); HB2, HB3 are the peripheral parts of hadron calonmeter barrel; $\mathrm{HCl}$ front/deep is parts of end cap 1 (the front part has two times higher sampling than the deep one); $\mathrm{HC} 2$ is end cap 2; EC is the electromagnetic end cap counter. 
Table 1

Weighting factors for different polar angles

\begin{tabular}{lllll}
\hline 0 & $42.3^{\circ}-54^{\circ}$ & $54^{\circ}-66^{\circ}$ & $66^{\circ}-78^{\circ}$ & $78^{\circ}-102^{\circ}$ \\
& $126^{\circ}-137.7^{\circ}$ & $114^{\circ}-126^{\circ}$ & $102^{\circ}-114^{\circ}$ & \\
\hline$W_{\text {BGO }}^{\prime J}$ & 1.42 & 1.40 & 1.39 & 1.40 \\
$W_{\mathrm{HC}}^{\prime \prime}$ & 1.07 & 1.09 & 1.12 & 1.10 \\
$W_{\mathrm{ee}}^{J J}$ & 1.02 & 1.02 & 1.01 & 1.00 \\
\hline
\end{tabular}

and $W_{1}$ is a weighting factor whose value is then obtained by minimizing the sum

$$
\sum_{\text {ll events }}\left(E_{\text {meas }}-E_{\mathrm{cm}}\right)^{2}
$$

where $E_{\mathrm{cm}}$ is the energy of the colliding electrons and positrons, corrected for inclusive muon energies as determined from the muon chambers. This minimization is done separately for $\mathrm{e}^{+} \mathrm{e}^{-}$and jet-jet events. Fig. $4 \mathrm{a}$ shows the distribution of the total number of hits and fig. $4 \mathrm{~b}$ the distribution of the front end (BGO) energy fraction; the separation illustrated here are used for electron-jet separation.

The result of the calibration procedure is a set of weighting factors $W_{t}$. Some of them are given in table 1 for illustration.

Hence, the standard L3 reconstruction procedure starts by the application of a pattern-recognition al-

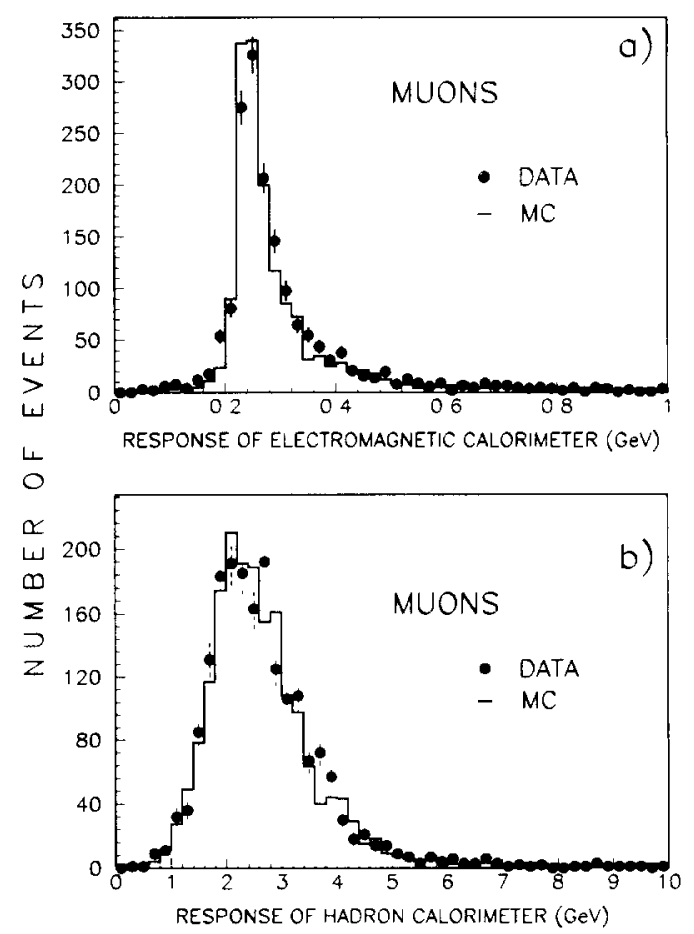

Fig. 5. Electromagnetic calorimeter response to muons (a) and hadron calorimeter response to muons (b).

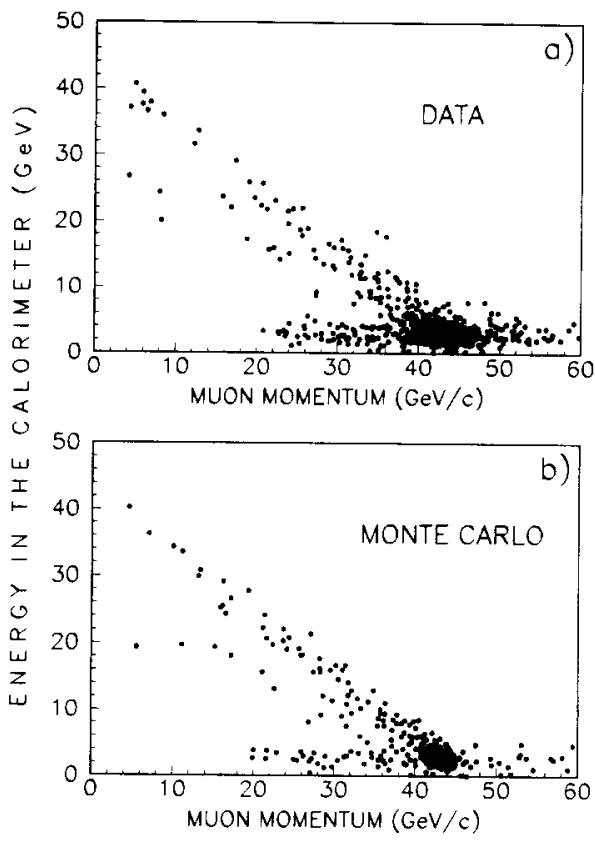

Fig. 6. The correlation of the muon momentum measured by the muon chambers and muon energy deposited in the hadron calorimeter for dimuon events in the data (a) and Monte Carlo (b).

gorithm. Then the energy deposited in each subdetector is calculated, using the weighting factors determined in the calibration, and the total energy of the event is determined.

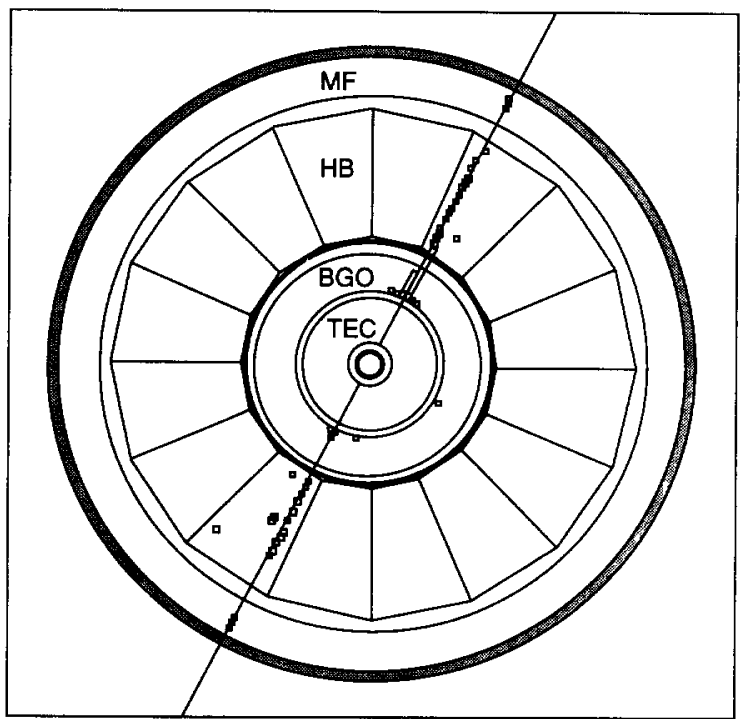

Fig. 7. Computer graph of a dimuon event in the calorimeter with a hard bremsstrahlung photon in BGO. 


\section{Results, $Z^{0}$ event analysis}

Data collected during 1989 and 1990 at energies at and near the $Z^{0}$ peak have been analysed using the pattern recognition and calibration algorithms described above. The data sample contains of the order of $10^{5}$ events. Cuts were applied to extract samples of electrons, muons, and hadron jets from the data.

\subsection{Muons}

The response to muons of the central electromagnetic and hadronic calorimeters is shown in figs. 5a and 5b, together with Monte Caerlo predictions. The long tail of events is due to the large energy loss undergone by the muons in the calorimeter through electromagnetic processes (bremsstrahlung, delta rays, etc.). This is llustrated in figs. $6 \mathrm{a}$ and $6 \mathrm{~b}$, where the calorimeter energy loss is plotted against the muon energy measured in the muon spectrometer. The correlation is clearly seen. The calorimeter information can thus improve the muon momentum measurement, in particular for energy losses of over $5 \mathrm{GeV}$ in the hadron calorimeter. Fig. 7 is the computer graph of a dimuon event in the detector
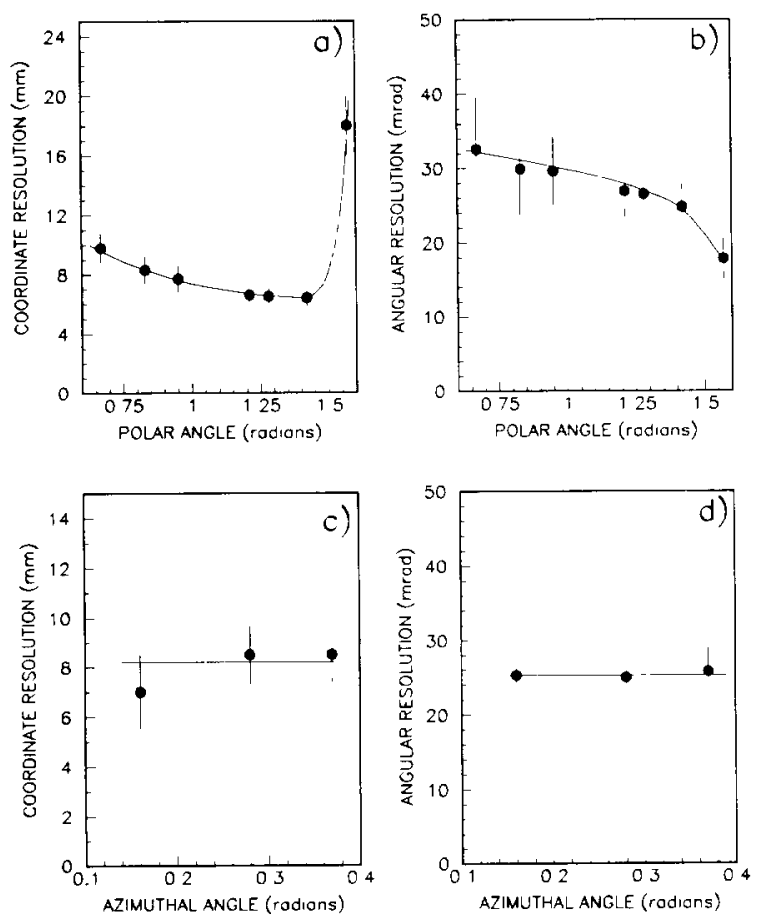

Fig. 8. Coordinate (a) and angular (b) reconstruction accuracy for muons as a function of polar angle $\theta$. Coordinate (c) and angular (d) reconstruction accuracy as a function of the azımuthal angle $\phi$. The angle of the incoming muon is measured with respect to normal incidence.

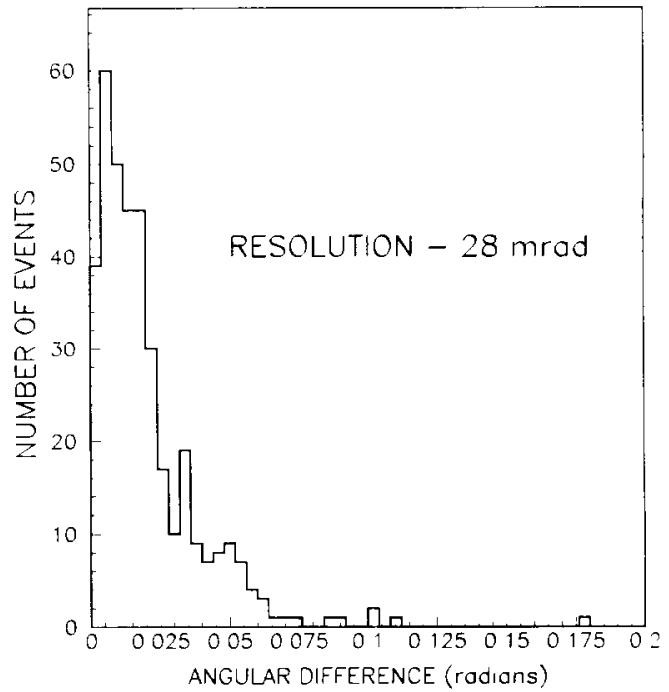

Fig 9. The difference between the $\theta$ angles measured by the muon chambers and by the hadron calorimeter

with a hard bremsstrahlung photon in the $\mathrm{BGO}$ calorimeter.

The single-track resolution of the $\mathrm{HC}$ barrel is illustrated in fig. 8 . These results, obtained with a muon test beam, show average values of 28 and $25 \mathrm{mrad}$ for the angular resolution in $\theta$ and $\phi$, respectively, and of about $8 \mathrm{~mm}$ for the transverse-coordinate resolution. The poorer coordinate resolution near $90^{\circ}$, seen in fig. $8 \mathrm{a}$, is due to the $\mathrm{HC}$ tower alignment there. In addition, from comparisons between the MF and the muon drift chambers, the muon filter is found to determine the coordinate of a muon on the $r-\phi$ plane with an accuracy of $2 \mathrm{~mm}$.

In fig. 9 the distribution of the difference in angle as measured by the muon chambers and the $\mathrm{HC}$ barrel for $\mathrm{Z}^{0} \rightarrow \mu \mu$ events is plotted. This distribution is in good agreement with test beam studies. Although the angular accuracy of the muon chambers is better than $1 \mathrm{mrad}$, there is multiple Coulomb scattering of about $3 \mathrm{mrad}$ in the calorimeter. In the off-line reconstruction, angular and coordinate information from the $\mathrm{HC}$ and the muon filter are used to resolve ambiguities in the muon chambers.

\subsection{Hadronic jets}

The response of the central portion of the calorimeter system to hadronic events is shown in fig. 10. The measured energy resolution is $10.2 \%$. The uniformity of the response over the fiducial volume is illustrated in fig. 11; the response is plotted as a function of the polar angle $\theta$ (fig. 11a) and azımuthal angle $\phi$ (fig. 11b) of the thrust axis. The mean response is uniform over the 


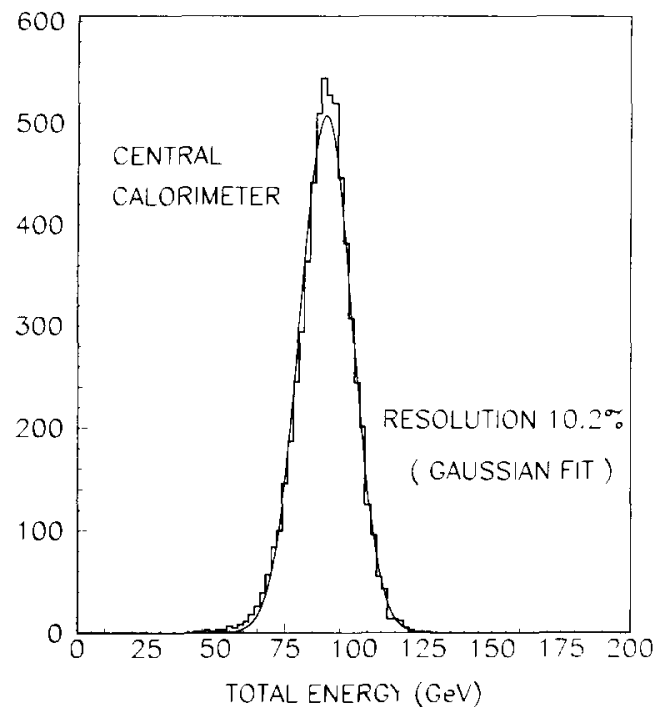

Fig. 10 The distribution of the total energy for two-jet hadronic events. Only events with both jets in the central part of the detector are plotted.

full angular range, although fluctuations are greater at both ends of the $\theta$ range. This effect is clearly seen in fig. 12, where the hadronic jet resolution is plotted as a
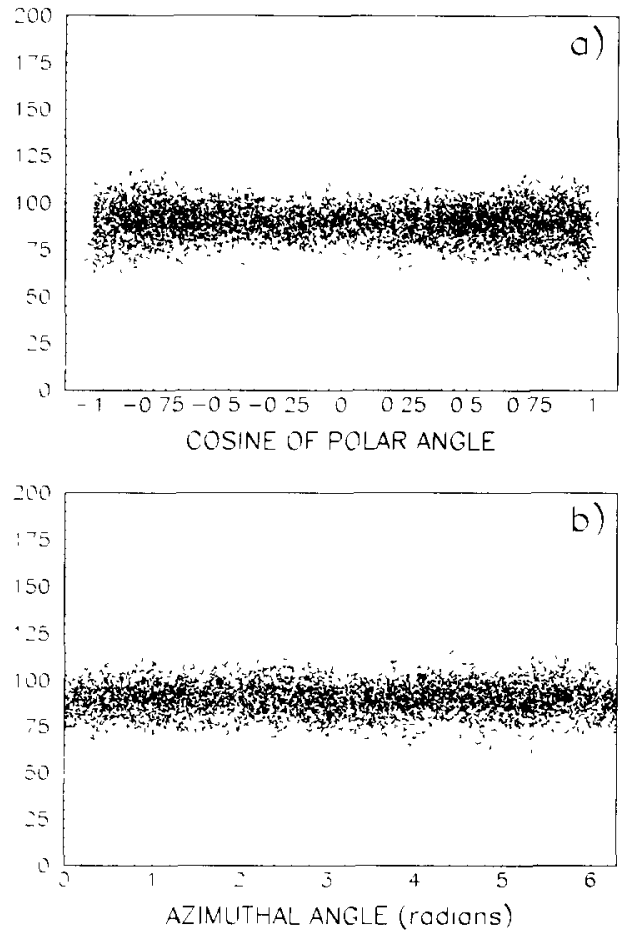

Fig 11 Uniformity of L3 detector response for hadronic events The total measured energy is plotted as a function of the angles $\theta$ (a) and $\phi$ (b) of the thrust axis.

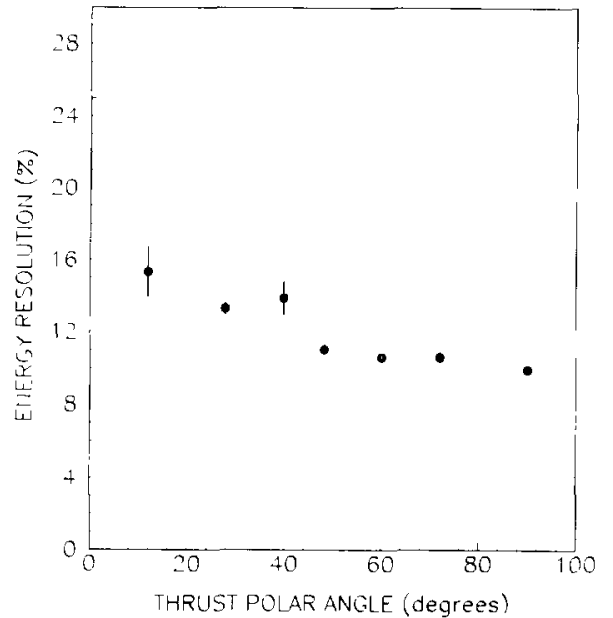

Fig. 12. The calorimeter energy resolution for hadronic events as a function of the angle $\theta$ of the thrust axis The resolution values in the forward and backward regions are discussed in the text

function of $\theta$. The resolution in the forward and backward regions is affected by leakages from the calorimeter near the beam axis, by the coarser sampling of the end caps, and by the incomplete absorption of electromagnetic showers in the veto counters.

The angular distribution of energy within a jet is shown in fig. 13, where the data and the Monte Carlo simulation are presented. The two-jet acollinearity angle for two-jet events is plotted in fig. 14. Also shown is a Monte Carlo simulation which agrees well with the data. Assuming the same accuracy for each jet, we obtain for the central region an accuracy of $37 \mathrm{mrad}$ in the determination of single-jet angles. Fig. 15 illustrates how the amount of energy found outside jets in a

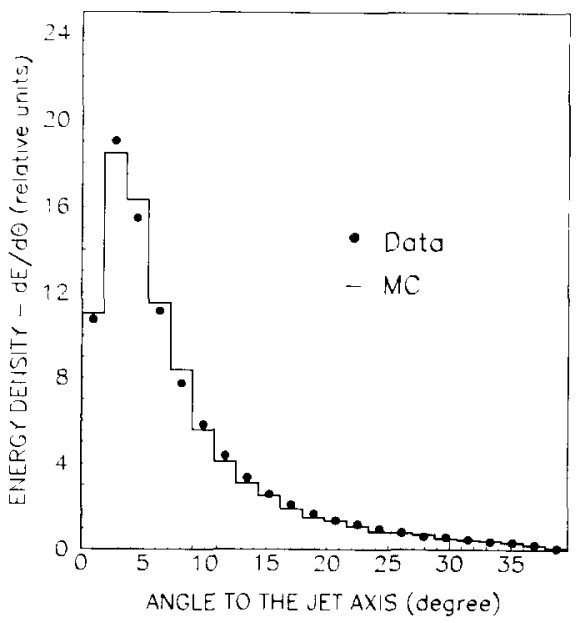

Fig. 13 Transverse-energy distribution inside a jet: $\theta$ is the angle with respect to the jet axis. 


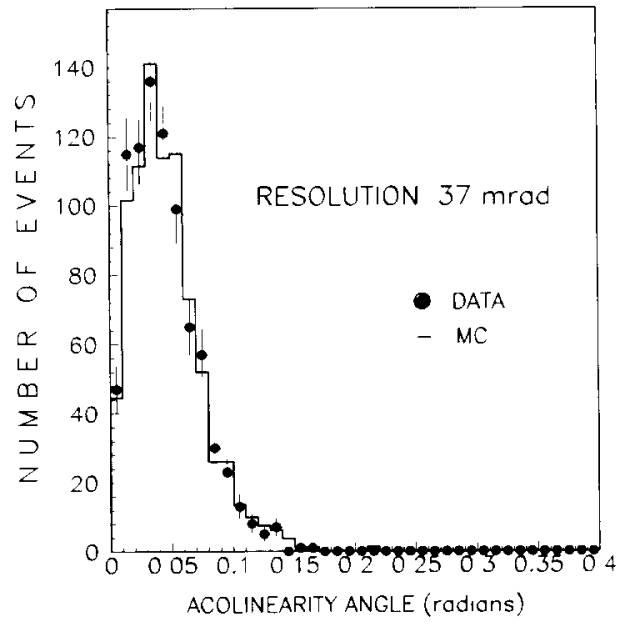

Fig. 14 Acollinearity angle for two-jet events.

two-jet event affects the determination of the jet angle. Naturally the less energy there is outside jets the better the jet direction is measured. The resolution levels off when less than $3 \mathrm{GeV}$ is outside the jets.

The computer graph of a typical two-jet $Z^{0}$ decay in the barrel is shown in fig. 16, that of an event in the end cap in fig. 17.

\section{Conclusions}

The L3 calorimeter has been operating successfully since August 1989. The long-term overall stability of the

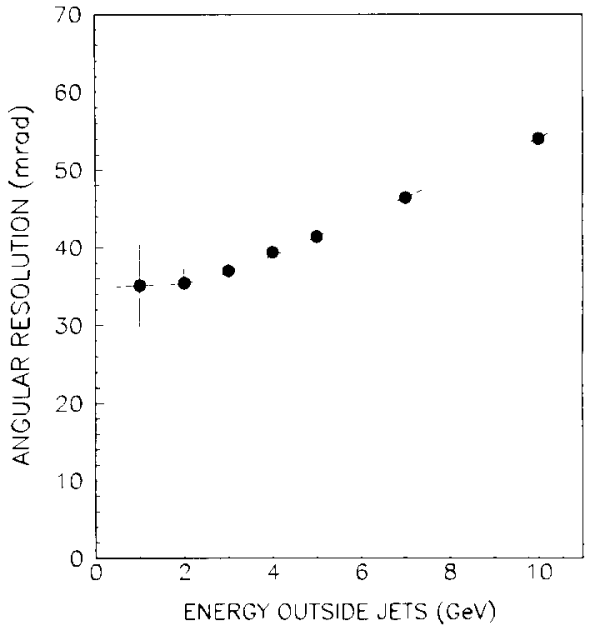

Fig. 15. Angular resolution determined from the sets of two-jet events with a different energy outside the jets.

readout, the electronics, and the chamber gain, is found to be better than $1.3 \%$. An algorithm has been developed to obtain uniformity of response over the entire solid angle covered by the calorimeter system and to minimize the error in jet energy measurement using two-jet events from $Z^{0}$ decays. The energy resolution obtained is $10.2 \%$ in the central region and $13.8 \%$ in the forward and backward regions of the system. Single hadron jet directıons are determıned to an accuracy of 37 mrad.

The correlation between the measured muon energy loss in the calorimeter and the muon momentum mea-

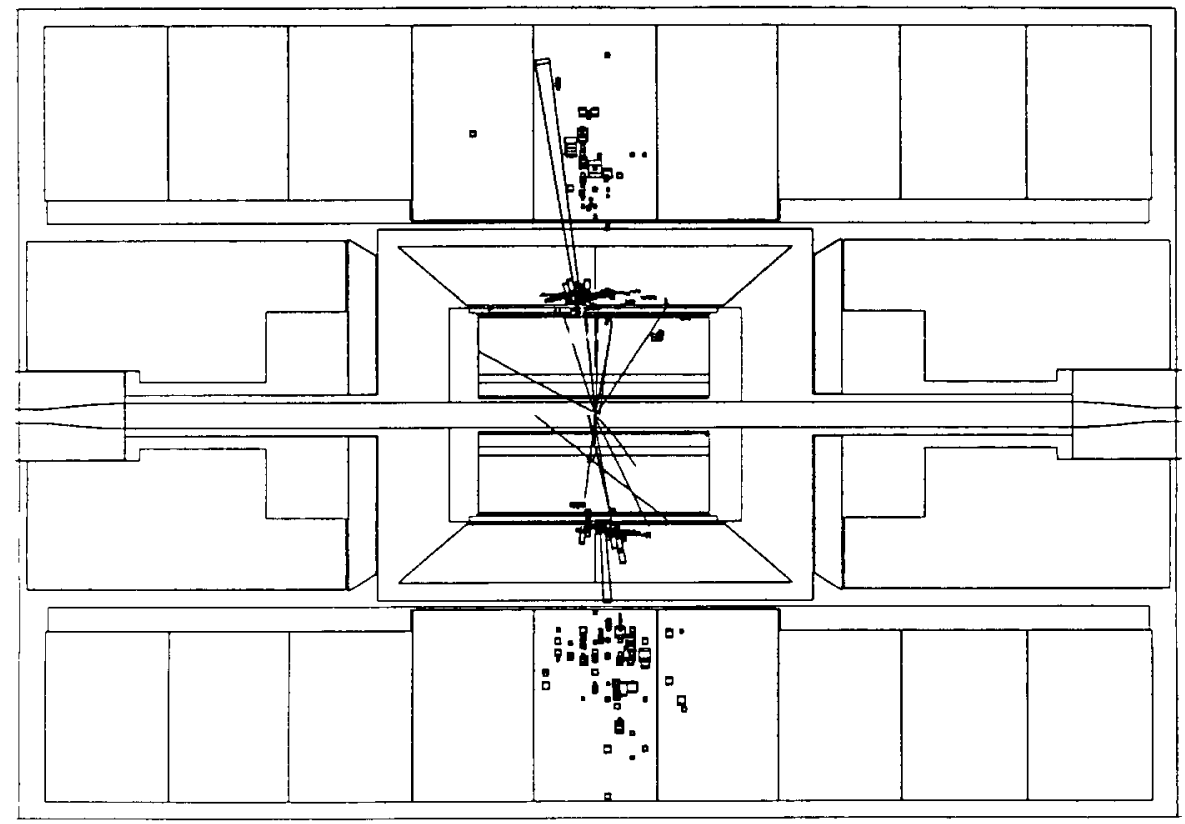

Fig. 16 The side view of a two-jet event in the L3 detector. 


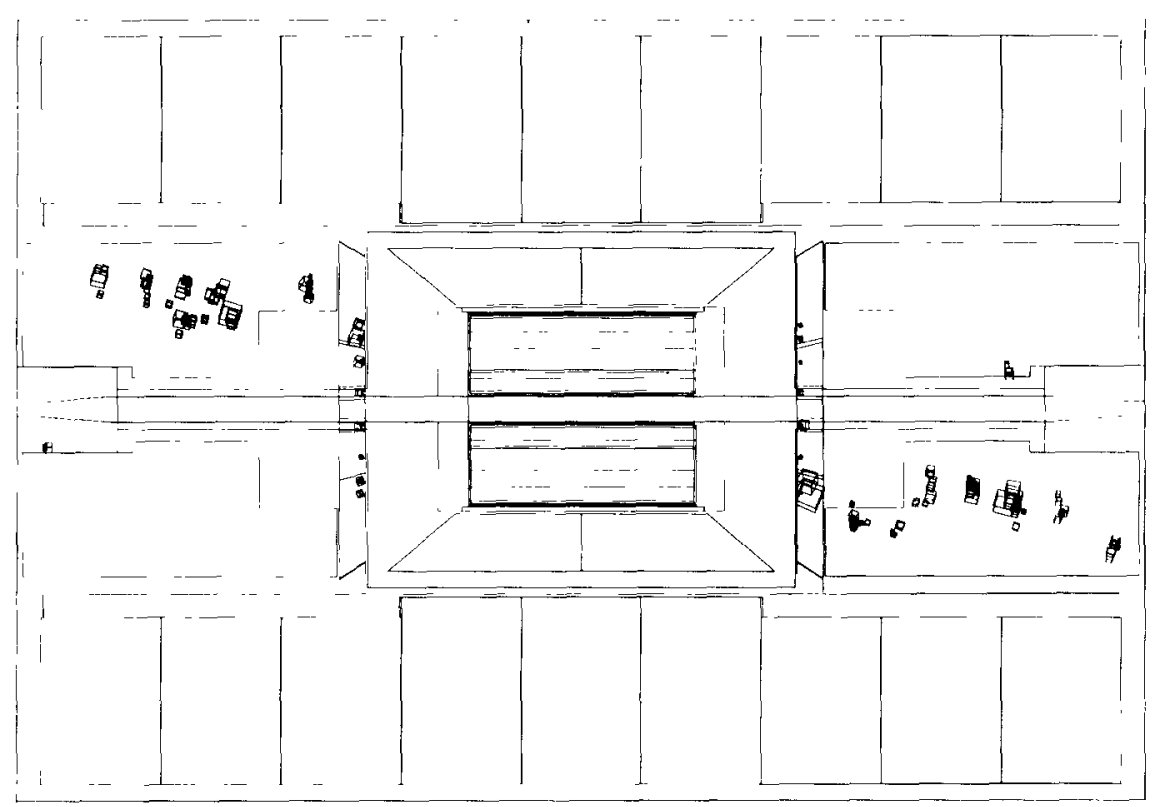

Fig 17. The side view of a two-jet event in the L3 end-cap.

sured in the muon chambers necessitates the use of the calorimeter data to improve the determination of muon momenta from the primary interaction by inclusion of large muon energy losses. The measurement of muon track position in the calorimeter is shown to provide a coordinate accuracy of about $8 \mathrm{~mm}$ and an angular accuracy of $25-28 \mathrm{mrad}$ in the barrel and $2 \mathrm{~mm}$ in the $r-\phi$ plane in the muon filter; these provide additional information for the global reconstruction of muons in the L3 detector.

\section{Acknowledgements}

We acknowledge the great effort made by the $\mathrm{BGO}$ group of the L3 Collaboration in the development of the algorithms used in the reconstruction of events in the BGO calorimeter, especially the contribution of Dr. D. Stickland. We are grateful to Dr. T. Hebbeker for useful discussions. The authors from the University of Michigan wish to acknowledge the support of the US National Science Foundation

\section{References}

[1] B. Adeva et al. (L3 Collab), Nucl. Instr. and Meth. A289 (1990) 35.

[2] S. Burov et al, preprint CERN-EP/88-84 (1988)

[3] A Arefiev et al, Nucl Instr and Meth. A285 (1989) 403.

[4] A Arefiev et al, Nucl Instr. and Meth A288 (1990) 364

[5] T. Sjostrand and M Bengtsson. Comput. Phys. Commun 43 (1987) 367:

T Sjostrand, in Z physics at LEP, rep CERN 89-08, Vol. 3 (CERN. Geneva, 1989) p 143

[6] GEANT Version 3.13 (September 1989); see R. Brun et al. GEANT 3, CERN rep CERN DD/EE/84-1 (revised in September 1987).

[7] B. Adeva et al. (L3 Collab.). Phys. Lett. B237 (1990) 136 